\title{
Leadership ist gefragt!
}

\begin{abstract}
Die Entwicklung hin zu agilen Organisationsformen, der Trend zu mehr Nachhaltigkeit sowie das immense Potenzial von Big Data und das durch die Digitalisierung ermöglichte Maß an Transparenz verstärken sich gegenseitig und stellen die Finanzfunktion vor große Herausforderungen. Prozessuale, instrumentelle und systemseitige Anpassungen allein greifen zu kurz. Für eine erfolgreiche Veränderung bedarf es Leadership, Vision und ein gerüttelt Maß an Energie, Frustrationstoleranz und Stehvermögen.
\end{abstract}

Utz Schäffer

Die durch die COVID-19-Pandemie geprägten vergangenen Monate haben vielleicht auch Ihnen die Gelegenheit gegeben, grundlegend über das nachzudenken, was sich seit der Finanzkrise in der Finanzfunktion und im Controlling geändert hat und was sich am Horizont für die nächste Dekade abzeichnet. Im folgenden Beitrag möchte ich das Zusammenspiel der großen Entwicklungslinien, den Status quo der Veränderung und die wesentlichen Implikationen für Finanzvorstände und Controlling-Leiter in einer Gesamtschau zusammenfassen.

\section{Zusammenfassung}

- Die Finanzfunktion kann in einer volatilen, unsicheren, komplexen und uneindeutigen Umwelt nur bestehen, wenn sie sich grundlegend verändert.

- Vier zentrale Entwicklungslinien prägen die Transformation: Agilität, Nachhaltigkeit, Big Data und Transparenz.

- Wer Veränderung als Residualgröße nach Erledigung des Tagesgeschäfts behandelt, wird letztlich scheitern. Es gilt, loszumarschieren und die Veränderung proaktiv zu gestalten.

\section{Entwicklungslinien}

Im Einzelnen unterscheide ich vier große Entwicklungslinien:

Erstens: Das organisatorische Grundprinzip in großen Unternehmen wechselt angesichts zunehmender Volatilität, Unsicherheit, Komplexität und Ambiguität der Umwelt vom bewährten Muster dominanter Plankoordination langsam, aber sicher zu etwas, was die Organisationstheorie „dominante Koordination durch Selbstabstimmung “nennt. Populärwissenschaftlicher formuliert: Netzwerke, agile Teams und das Prinzip des Experiments ersetzen zunehmend Hierarchie und Plan. Diesem Trend kann sich auch die Finanzfunktion nicht entziehen. Gerade für Controller ist diese Entwicklung von ganz zentraler Bedeutung, da sie traditionell in einem Kontext dominanter Plankoordination beheimatet sind. Controller arbeiten in einem Umfeld, in dem Pläne dominieren und der kybernetische Regelkreis in Form eines Systems formaler Planungs- und Kontrollprozesse institutionalisiert ist. In kleinen Unternehmen, die dominant durch die Weisung des Chefs gesteuert werden, in Forschungs- und anderen Teams, die sich selbst abstimmen, und im Kontext programmkoordinierter Bürokratie sind Controller (die diesen Namen auch verdienen) hingegen kaum zu finden. Kein Wunder also, dass sich Controller mit dem Thema Agilität 
schwertun. Ihre Denkmuster und Routinen sind eng mit der Plankoordination verbunden. Müssen sich Controller dennoch organisatorisch neu erfinden und ihr Mindset im Zuge eines Trends zu agilen Formen des Managements grundlegend verändern?

Zweitens: Die Prinzipien von ökonomischer, sozialer und ökologischer Nachhaltigkeit sowie das aktuell viel diskutierte Konzept der Resilienz stellen die dominante Ausrichtung des Handelns an Effizienz und kurzfristiger Maximierung von Rendite und Börsenwert infrage. Für beides stehen Finanzvorstände und Controller wie kaum ein anderer Akteur im Unternehmen. In den Schriften mancher Vordenker und in der gesellschaftlichen Diskussion wird der kurzfristigen Maximierung von Effizienz, Rendite und Börsenwert mit den Konzepten der Nachhaltigkeit und Resilienz aber zunehmend eine Denkweise gegenübergestellt, die wieder den eigentlichen Zweck des unternehmerischen Handelns („Purpose“), langfristiges Denken und das Prinzip des rechten Maßes in den Mittelpunkt rückt. Was aber heißt das für Finanzvorstände und Controller? Sollen sie sich gegen diesen Trend oder an die Speerspitze der Entwicklung stellen? Muss sich etwas verändern - im Denken, Handeln und Reden?

Drittens: Die Digitalisierung und das Potenzial von Big Data stellen infrage, ob Small Financial Data und das damit verbundene Handwerkszeug als traditionelle Basis des Controllings ausreichen, um Manager auch in Zukunft als „Single Source of Truth" und Business Partner erfolgreich zu begleiten. Der Aufstieg der Controller-Profession vor 30 bis 50 Jahren beruhte in erster Linie darauf, dass Controller Gewinn, Kosten und - eingeschränkt - Erlöse besser analysieren konnten als die meisten Manager und damit eine wichtige Brücke zwischen Leistungserstellung und Finanzwelt darstellten. Was, wenn mittlerweile gerade in börsennotierten Unternehmen der Kontakt der Finanzfunktion zur zugrunde liegenden Realwirtschaft brüchig geworden ist? Was, wenn die Verbindung von finanziellen und vorlaufenden nichtfinanziellen Kennzahlen zunehmend an Bedeutung gewinnt und das reine Nebeneinanderstellen entsprechender Schlüsselgrößen in einem Bericht keine ausreichende Antwort darstellt? Was, wenn die neue Währung zunehmend Big Data ist und selbstlernenden Algorithmen eine immer wichtigere Rolle zukommt? Was, wenn Data Scientists und andere Berufsgruppen große, schlecht strukturierte Mengen nichtfinanzieller Daten besser analysieren können und Grenzen und Möglichkeiten der Künstlichen Intelligenz besser verstehen als Controller? Reicht es dann aus, sich als Gralshüter der Finanzen und als Brücke zum Management zu verstehen, oder stellen sich weitergehende Fragen?

Viertens: Die Digitalisierung ermöglicht neben ungeahnten Effizienzfortschritten und neuen Möglichkeiten der Datenanalyse auch ein deutlich höheres Maß an intraorganisationaler Transparenz. Dieses wird im Kontext agiler Organisationsformen auch zunehmend benötigt und von der nachwachsenden Generation eingefordert. Ein Mehr an Transparenz hat aber weitreichende Implikationen. Zum einen steigen die Anforderungen an die Qualität von Daten dramatisch an. Zum anderen werden traditionelle Ausprägungen der Data Governance mit ihrem Fokus auf das Management eines komplexen Systems von Zugriffsberechtigungen grundlegend infrage gestellt. Die Entwicklung erzwingt auf Sicht eine weitgehende Demokratisierung des Informationszugangs und eine Kultur des offenen funktions-, hierarchie- und siloübergreifenden Informationsaustauschs. Auch dieser Trend hat somit enorme Implikationen für das Selbstverständnis von Controllern, die ihre traditionelle Rolle als Gatekeeper für betriebswirtschaftliche Zahlen und Single Source of Truth entsprechend an veränderte technische und kulturelle Rahmenbedingungen anpassen müssen.

\section{„Es geht eben nicht um einzelne Projekte oder um eine neuartige Systemlösung, sondern um eine grundlegende Verände- rung der Finanzfunktion."}

Alle vier skizzierten Entwicklungen haben grundlegende Implikationen für Unternehmenssteuerung und Controlling. Sie ergänzen und verstärken sich dabei zumindest in Teilen gegenseitig. Die Digitalisierung ermöglicht neue Formen der Datenanalyse und mehr Transparenz, diese ist wiederum eine wichtige Voraussetzung für mehr Agilität. Und Agilität spielt nicht nur im Kontext der digitalen Transformation, sondern auch im Konzept der Resilienz eine tragende Rolle. Für Finanzvorstände und Controlling-Leiter ist dies eine wichtige Einsicht, weil - folgt man dem Gedanken - es eben nicht um eine mehr oder weniger große Anzahl einzelner Projekte oder um eine neuartige Systemlösung geht, sondern um verschiedene Facetten einer grundlegenden Veränderung der Finanzfunktion, an deren möglichem Ende ein neuer eingeschwungener Zustand stehen könnte. Gleichzeitig stehen die meisten Unternehmen heute noch eher am Anfang der von den genannten Veränderungen ausgelösten Transformation. 


\section{Status quo}

Wenden wir uns zunächst dem durch die veränderte Umwelt induzierten Wandel von Strukturen und Prozessen zu. Die Notwendigkeit, tradierte Gestaltungsmuster zu hinterfragen und die Organisation an einen Kontext hoher Volatilität, Unsicherheit, Komplexität und Ambiguität anzupassen, wird in der Finanzfunktion entweder immer noch ignoriert oder auf die Verschlankung oder marginale Anpassung bereits bestehender Prozesse und eine Handvoll mehr oder weniger ,agiler" Veränderungsprojekte reduziert. Selbst bei den Pionieren steht eine agile Transformation der Finanzfunktion häufig noch in ihren Anfängen, und es besteht ein hohes Maß an Unsicherheit, wie weit Veränderungen gehen sollen. Während sich Management und Finanzfunktion so an neue Organisationsformen und die damit verbundenen Techniken und Denkmuster herantasten, bleibt der überwiegende Teil der Bestandsorganisation in Unternehmen unverändert plankoordiniert. Die damit verbundenen Routinen in Finanzfunktion und Unternehmenssteuerung sowie das viel zitierte Mindset werden nicht grundsätzlich infrage gestellt. In einem vergleichsweise stabilen Kontext macht dieses Vorgehen durchaus Sinn und entspricht zudem den etablierten Denkmustern. Es stößt aber an Grenzen, wenn es darum geht, die Innovationsund Anpassungsfähigkeit der Organisation auch mithilfe der Finanzfunktion substanziell und schnell zu erhöhen. Zahlreiche Unternehmen der deutschen Automobil- und Finanzindustrie können bereits ein Lied davon singen. Und sie sind nicht die einzigen.

\section{„Vieles spricht dafür, dass sich das notwendige Maß an Resilienz und Nachhaltigkeit weiter erhöhen wird. "}

Ein noch deutlicheres Bild ergibt sich mit Blick auf die Konzepte der Nachhaltigkeit und der Resilienz. Beide werden weithin immer noch primär als Marketing-Instrumente und/ oder regulatorische Nebenbedingung betrachtet, weniger als eine grundsätzliche Veränderung des Denkens. Und ähnlich wie im Fall der organisatorischen Veränderung war auch diese Praxis bislang durchaus nicht irrational. Präziser formuliert: Sie entspricht dem vorherrschenden Verständnis von Rationalität, nach dem Unternehmen nur dann in Resilienz oder Ökologie investieren können, wenn sich das auch kurzbis mittelfristig rechnet oder Gesellschaft und Kapitalmarkt gewisse Mindeststandards einfordern. Ein Mehr an Nachhaltigkeit ist also (nur) erlaubt, wenn sich ein nachhaltiges Produkt profitabler verkaufen lässt, wenn sich eine Sparmaßnahme unter Verweis auf die Ökologie besser begründen lässt, wenn ein soziales oder ökologisches Image die Kundenbindung messbar steigert oder wenn sich die gesetzlichen Anforderungen erhöhen. Doch langsam scheint sich etwas zu ändern: Die Fridays-for-Future-Bewegung, die Forderungen des einen oder anderen Großinvestors und der Auftritt mancher Vorstandssprecher beim Weltwirtschaftsforum in Davos signalisieren, dass der aktuelle Umgang mit dem Thema der ökologischen Nachhaltigkeit die gesellschaftliche Betriebserlaubnis auf Dauer gefährden könnte. Und die aktuelle CoronaPandemie wirft die Frage auf, ob und wie Unternehmen ihre ökonomische Nachhaltigkeit und Resilienz in einem Kontext immer wiederkehrender externer Schocks erhöhen können. Vieles spricht also (auch betriebswirtschaftlich) dafür, dass sich das notwendige Maß an Resilienz und Nachhaltigkeit weiter erhöhen wird, das Denken langfristiger werden sollte und eine weitgehende Reduktion des Themas auf Marketing und Compliance immer weniger ausreicht. Wenn dem aber so ist, betrifft das auch die Finanzfunktion: Sie muss sicherstellen, dass auf die hehren Sprüche in Davos sowohl bei Investitionsentscheidungen als auch im Alltag der Unternehmenssteuerung Taten folgen.

Wenden wir uns dem dritten Trend zu: Die Analyse von Big Data und die damit verbundenen Fähigkeiten werden trotz des Digitalisierungs-Hypes der vergangenen Jahre in aller Regel nicht als zentrale Fähigkeit eines Controllers betrachtet und entsprechend ist die diesbezügliche Expertise in den meisten Finanzbereichen eher überschaubar. Viele Führungskräfte in der Finanzfunktion gehen davon aus, dass die traditionellen Unterstützungs- und Rollenmuster Bestand haben und Controller zukünftig als Brücke zwischen dem Management und auf Big Data spezialisierten Analysten fungieren werden, grundlegende Veränderungen also nicht erforderlich sind. Ob sie recht haben, wird die Zukunft zeigen. Dagegen spricht, dass auch für die Wahrnehmung einer Übersetzungsund Brückenfunktion ein solides Grundwissen bezüglich der Analyse großer und gegebenenfalls auch wenig strukturierter Datenmengen sowie eine profunde Expertise in der Verbindung von finanziellen und nichtfinanziellen Kenngrößen unabdingbar sind. Andere Funktionen wie etwa das Marketing oder das Supply Chain Management sind der Finanzfunktion hier oft ein gutes Stück voraus. Zudem entstehen schon heute neue Unterstützungsrollen rund um die Themen Daten- 
Management und Datenanalyse, und auch das Rollenbündel der Controller entwickelt sich zumindest im Kontext des einen oder anderen großen Unternehmens weiter. Gleichzeitig können wir beobachten, dass traditionelle ScorekeeperAufgaben der digitalisierungsinduzierten Automatisierung zunehmend zum Opfer fallen und erste engagierte Analytiker und Manager in Data Science Teams langsam in die Rolle einer Brücke zum Management hineinwachsen, sich eher als entscheidungs- und aktionsorientierter Decision Scientist denn als Rechenknecht für Big Data sehen.

Ein weiterer Aspekt ist nicht zu vernachlässigen: Der Zusammenhang zwischen finanziellen Steuerungsgrößen und nichtfinanziellen Variablen ist immer geschäftsspezifisch. Wer auf der Ebene eines Geschäftsmodells datengetrieben steuern will, muss deshalb fundierte Geschäftskenntnisse besitzen. Bleibt das zugrunde liegende Geschäft über viele Jahre im Wesentlichen unverändert, kann sich genügend gemeinsames Wissen aufbauen, um eine weitgehend separate Finanzperspektive zu ermöglichen. In einer durch Volatilität, Unsicherheit, Komplexität, Ambiguität und nicht zuletzt die starke Veränderung von Geschäftsmodellen geprägten Welt ist dies aber hochgradig problematisch. Schnell findet die finanzielle Steuerung in einer Scheinwelt statt. Finanzvorstände und Controller müssen daher stets die Geschäfte im Detail kennen und auch deren Veränderungen unmittelbar begleiten. Dann und nur dann sind sie in der Lage, den Zusammenhang zwischen vor- und nachlaufenden Steuerungsgrößen zu beherrschen und erfolgreich als Brücke zwischen dem Management und der Analyse von Big Data zu fungieren. In den vergangenen Jahren konnte man aber bisweilen den Eindruck gewinnen, dass die finanzielle Steuerung in manchen Unternehmen eher ein Eigenleben führt und der Kontakt zur zugrunde liegenden Realwirtschaft nicht mehr vollumfänglich belastbar ist.

Werfen wir abschließend noch einen Blick auf den vierten Trend, die Entwicklung hin zu mehr Transparenz. Zahlreiche Unternehmen sind vollumfänglich damit beschäftigt, im Maschinenraum die Voraussetzungen für mehr Transparenz zu schaffen, das heißt ihre Systemlandschaften zu harmonisieren und die Defizite in der Data Governance durch aufwendige Projekte zu beseitigen. Aber noch bewirkt die Vision voll integrierter Systemlandschaften oder auch das Bild eines umfassenden „Data Lakes“ im Regelfall das gleiche müde Lächeln wie das Versprechen, dass manuelle Brückenarbeiten in Excel überflüssig werden. Und auch die zahlreichen auf mehr „Self-Service“ im Management zielenden Pilotprojekte resultieren eher selten in einem weitgehend de- mokratisierten Informationszugang. Gelegentlich ist sogar zu beobachten, wie im Zuge solcher Projekte bereits erweiterte Zugangsberechtigungen wieder revidiert werden. Der Status quo der Informationsversorgung des Managements steht damit trotz schöner Frontend-Lösungen in einem gewissen Spannungsverhältnis zum Postulat der meisten neueren Management-Konzepte, die in einem Kontext ständiger Veränderung auf dezentrale Initiative und das „Empowerment“ sich flexibel zusammensetzender Teams setzen. Hier stoßen veraltete Systemlandschaften und eng gefasste Zugangsberechtigungen genauso an Grenzen wie ein überkommenes funktionales Mindset. Eine hinreichende Transparenz ist die notwendige Voraussetzung für mehr dezentrale Initiative in einem Kontext hoher Veränderung.

\section{„Transparenz ist die notwendige Voraus- setzung für mehr dezentrale Initiative."}

Mit Blick auf alle vier Entwicklungen gilt es also festzuhalten, dass die Unternehmenspraxis in aller Regel noch eher am Anfang der postulierten Transformationspfade steht und dass inkrementelle Veränderungen im gegebenen Rahmen von tradierten Rollen und Strukturen dominieren. Das ist nicht verwunderlich. Zum einen erfordert die grundlegende Veränderung von Systemlandschaften, Strukturen, Routinen und tief verankerten Denkmustern in bestehenden Organisationen viel Zeit: Jahre, vielleicht auch eine ganze Generation von Managern und Controllern. Zu groß sind die Beharrungskräfte auf der einen und der Veränderungsbedarf auf der anderen Seite. Zum anderen stehen Finanzvorstände und ControllingLeiter heute mehr denn je unter einem enormen Effizienzdruck, der Veränderung und Experiment scheinbar nur in wohldefinierten Nischen und im Rahmen von etablierten Organisations- und Rollenmodellen sowie weitgehend gegebener Kultur möglich macht.

\section{Implikationen}

Veränderungsprojekte innerhalb eines durch Struktur und Kultur geprägten Rahmens mögen ein guter Ansatz sein, um die genannten Herausforderungen trotz kurzfristigen Erfolgsdrucks anzugehen und die Organisation überhaupt in Bewegung zu versetzen. Um das Unternehmen wirklich agil, nachhaltig und resilient zu machen, um das Potenzial der Digitalisierung wirklich in vollem Umfang zu heben, reichen instrumentelle und prozessuale Veränderungen in einem ge- 
gebenen Kontext auf Dauer aber nicht aus. Sie stoßen (sehr) schnell an Grenzen - auch und gerade mit Blick auf die Finanzfunktion und den Prozess der Unternehmenssteuerung.

Die verschiedenen Bausteine der Steuerung - Informations-, Planungs-, Kontroll- und Anreizsysteme, aber eben auch Organisation und Kultur - greifen ineinander und müssen konsistent und aufeinander abgestimmt gestaltet werden. Bei entsprechendem Veränderungsbedarf ist die Veränderung der Unternehmenskultur und der mit ihr verbundenen Denkmuster zumindest auf Dauer unabdingbar und gleichzeitig in aller Regel die zentrale Barriere im Transformationsprozess: „Culture eats strategy for breakfast", wusste Peter F. Drucker schon früh zu berichten. Warum ist das so? Tradierte Denkmuster und eine fest im Unternehmen verankerte Kultur sind träge. Es bedarf viel Energie, sie zu verändern, und wie wir aus der Literatur zum Veränderungs-Management lernen können, wird ihre Mobilisierung in der Regel nur erfolgreich sein, wenn die Dringlichkeit der Veränderung intellektuell und emotional verstanden wurde, sei es im positiven Sinne die sich bietenden Möglichkeiten lassen keinen anderen Weg $\mathrm{zu}$ - oder im negativen Sinne - wenn wir diesen Weg nicht gehen, ist unsere Existenz gefährdet.

\section{„Die Veränderung der Unternehmens- kultur und der mit ihr verbundenen Denkmuster ist unabdingbar im Transformationsprozess."}

Ein zweiter Aspekt kommt hinzu: Das gewünschte Maß an Veränderung lässt sich nicht beliebig portionieren. Die Entwicklung von Kultur und Mindset beinhaltet Schwelleneffekte, das heißt, es gibt so etwas wie eine kritische Masse an notwendiger Veränderung. So lassen sich 20 Prozent agil und 80 Prozent bürokratisch vielleicht organisatorisch abbilden, kulturell stößt eine solche Lösung aber an Grenzen und droht, das zarte Pflänzchen Agilität im Keim oder spätestens im nächsten Winter ersticken zu lassen. Daran ändert auch das an dieser Stelle gelegentlich bemühte Konzept der Ambidexterität nichts. Inselprojekte und primär instrumenten- und prozessorientierte Ansätze bleiben in der Regel weit von den kritischen Schwellenwerten der Kulturveränderung entfernt und sind genau deshalb am Ende so oft zu Wirkungslosigkeit und Scheitern verurteilt. Um es nochmals ganz deutlich zu sagen: In einem vergleichsweise stabilen Umfeld ist das wenig dramatisch, in dem Maße, wie die Einschätzung einer ganz grundlegenden Veränderungsnotwendigkeit trägt, aber schon.

Das Dilemma, vor dem sich viele Führungskräfte sehen, erscheint damit offensichtlich: Die Transformation der Finanzfunktion lässt sich nicht auf prozessuale, instrumentelle oder systemseitige Anpassungen reduzieren, und die notwendige kulturelle Veränderung benötigt viel Energie und Zeit, die aber im Regelbetrieb nicht zur Verfügung stehen. Was also tun? Die Beyond Budgeting Community, die sich seit rund 20 Jahren mit der Frage befasst, welche Implikationen eine zunehmende Volatilität, Unsicherheit, Komplexität und Ambiguität der Umwelt auf Management und Unternehmenssteuerung haben, ist von Kindesbeinen an durch die Diskussion geprägt, in welcher Reihenfolge Steuerungsprozesse auf der einen und Organisation und Kultur auf der anderen Seite verändert werden sollten. Schon im Entstehungsprozesses des Bestsellers von Jeremy Hope und Robin Fraser von 2003 hatten Autoren und Verlag an dieser entscheidenden Stelle unterschiedliche Vorstellungen, und auch seitdem ist die Entwicklung der Community von der Diskussion geprägt, ob man die beiden großen Bausteine gleichzeitig oder in der einen oder anderen Reihenfolge adressieren sollte. Das Problem ist - wenig überraschend - bis heute nicht abschließend gelöst, weder im Kontext des Beyond Budgeting noch an anderer Stelle. Aber der (vermeintlich) pragmatische Ansatz, dominant auf die Prozessebene zu setzen, scheint empirisch doch klar zu überwiegen. Und damit schließt sich der Kreis und wir stehen wieder am Anfang dieses Absatzes.

Was heißt das aber nun für Führungskräfte in der Finanzfunktion? Ich meine: Leadership ist gefragt! Weil die oben skizzierten Herausforderungen ineinandergreifen, alle Branchen betreffen und sich gegenseitig verstärken, ist der Veränderungsbedarf in der Regel groß. Und wenn dem so ist, wird eine Transformation, die Veränderung als Residualgröße nach Erledigung des Tagesgeschäfts behandelt, letztlich scheitern. Der zuvor beschriebene Weg inkrementeller Veränderungen innerhalb des gegebenen Rahmens greift zu kurz, und kulturelle Versäumnisse lassen sich nicht „bei Bedarf“ schnell aufoder nachholen. Es gilt also, loszumarschieren und die Veränderung der Finanzfunktion als Change oder Transformation Agent proaktiv zu gestalten und dabei nicht zuletzt die eigene Funktion im Sinne eines „Role Makings“ weiterzuentwickeln. Ohne echtes Leadership, ohne Vision und ein gerüttelt Maß an Frustrationstoleranz, Rückgrat und vor allem auch Energie und Stehvermögen wird das aber nur in wenigen Fällen gelingen. Führungsqualitäten werden so zum kritischen 
Engpass der Transformation. Wichtig ist, dass Finanzvorstände und Controller das verstehen und sich nicht auf finanzwirtschaftliche Expertise und Kapitalmarktorientierung reduzieren lassen. Dabei müssen sie sich neben dem intensiven Bemühen um ein besseres Verständnis des zugrunde liegenden Geschäfts und neuer Technologien auf ihre ureigenen Stärken besinnen, nicht zuletzt auf die Fähigkeit, durch zahlen- und datenbasierte Analysen und eine Rolle als kritischer Counterpart die Rationalität des Managements auch und gerade in Zeiten der Veränderung sicherzustellen. Die Rahmenbedingungen und die Herausforderungen ändern sich, dieser zentrale Kern ihrer Aufgabe bleibt unverändert und ist wichtiger denn je.

Dieser Beitrag beruht zum Teil auf Überlegungen, die bereits in früheren Veröffentlichungen von mir publiziert wurden, einige davon in Co-Autorenschaft mit Jürgen Weber. Für wertvolle Hinweise und Anregungen danke ich Lars Brückner, Christian Bungenstock, Björn Radtke und Marko Reimer.

\section{Angaben zum Autor}

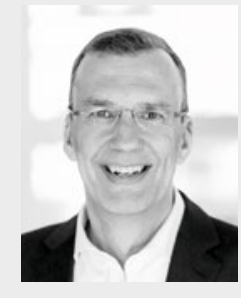

\section{Prof. Dr. Utz Schäffer}

ist Direktor des Instituts für Management und Controlling (IMC) der WHU - Otto Beisheim School of Management, Vallendar, und Mitherausgeber der Controlling \& Management Review.

E-Mail: utz.schaeffer@whu.edu

\section{SpringerProfessional}

Veränderung Finanzfunktion

Dahm, M. H./Thode, S. (Hrsg.) (2020): Digitale Transformation in der Unternehmenspraxis. Mindset - Leadership - Akteure Technologien, Wiesbaden.

www.springerprofessional.de/link/18289508

Harwardt, M./Niermann, F.-J./Schmutte, A. M./Steuernagel, A. (Hrsg.) (2020): Führen und Managen in der digitalen Transformation - Trends, Best Practices und Herausforderungen, Wiesbaden. www.springerprofessional.de/link/17962950

Feldbauer-Durstmüller, B./Mayr, S. (Hrsg.) (2019): Controlling - Aktuelle Entwicklungen und Herausforderungen. Digitalisierung, Nachhaltigkeit und Spezialaspekte, Wiesbaden www.springerprofessional.de/link/17134730

Schäffer, U./Weber, J. (2019): Zehn Thesen zur Entwicklung des Controllings, in: Controlling \& Management Review, 63 (6), S. 46-49. www.springerprofessional.de/link/17124712

\section{Springer Gabler}

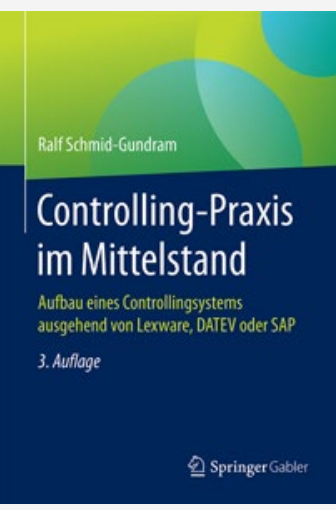

R. Schmid-Gundram

Controlling-Praxis

im Mittelstand

Aufbau eines

Controllingsystems

ausgehend von Lexware, DATEV oder SAP

3. Aufl. 2020. X, 234 S. 86 Abb.

Brosch.

$€$ (D) $37,99 \mid €$ (A) $39,06 \mid$ CHF 42.00

ISBN 978-3-658-28623-1

$€$ (D) 29.99 | CHF 33.50

ISBN 978-3-658-28624-8 (eBook)

- Anleitung zur Konzeption eines Controlling-Systems im

Mittelstand unter

Berücksichtigung spezieller Branchen

- Praxisnähe durch zahlreiche Umsetzungsbeispiele für Lexware, DATEV und SAP

- Dient der profitablen und zukunftssicheren Steuerung und Ausrichtung des Unternehmens mit Berücksichtigung von Business Intelligence

$€(D)$ sind gebundene Ladenpreise in Deutschland und enthalten $7 \%$ für Printprodukte und für elektronische Produkte. $€$ (A) sind gebundene Ladenpreise in Österreich und enthalten $10 \%$ für Printprodukte bzw. 20\% MuSt für elektronische Produkte. Die mit * gekenn-zeichneten Preise sind unverbindliche Preis-empfehlungen und enthalten die landesübliche MwSt. Preisänderungen und Irrtümer vorbehalten.

Part of SPRINGER NATURE springer.com/empfehlung 